\title{
Many Faces of Expertise: Fusiform Face Area in Chess Experts and Novices
}

\author{
Merim Bilalić, ${ }^{1}$ Robert Langner, ${ }^{2,3}$ Rolf Ulrich, ${ }^{4}$ and Wolfgang Grodd ${ }^{2}$ \\ ${ }^{1}$ Department of Neuroradiology, University of Tübingen, 72076 Tübingen, Germany, ${ }^{2}$ Department of Psychiatry, Psychotherapy and Psychosomatics, \\ Medical School, RWTH Aachen University, 52056 Aachen, Germany, ${ }^{3}$ Institute of Neuroscience and Medicine, Research Centre Jülich, 52428 Jülich, \\ Germany, and ${ }^{4}$ Department of Psychology, University of Tübingen, 72070 Tübingen, Germany
}

The fusiform face area (FFA) is involved in face perception to such an extent that some claim it is a brain module for faces exclusively. The other possibility is that FFA is modulated by experience in individuation in any visual domain, not only faces. Here we test this latter FFA expertise hypothesis using the game of chess as a domain of investigation. We exploited the characteristic of chess, which features multiple objects forming meaningful spatial relations. In three experiments, we show that FFA activity is related to stimulus properties and not to chess skill directly. In all chess and non-chess tasks, experts' FFA was more activated than that of novices' only when they dealt with naturalistic full-board chess positions. When common spatial relationships formed by chess objects in chess positions were randomly disturbed, FFA was again differentially active only in experts, regardless of the actual task. Our experiments show that FFA contributes to the holistic processing of domain-specific multipart stimuli in chess experts. This suggests that FFA may not only mediate human expertise in face recognition but, supporting the expertise hypothesis, may mediate the automatic holistic processing of any highly familiar multipart visual input.

\section{Introduction}

Recognizing human faces is one of the most essential visual skills — and also one of the most practiced ones. Since the very beginning of our lives, we have been exposed to faces as a major source of social information. The neural substrates of face recognition have been extensively studied (Kanwisher et al., 1997; Gauthier and Nelson, 2001; Rotshtein et al., 2005; Xu, 2005; Yovel and Kanwisher, 2004, 2005; Yue et al., 2006). One of the most important brain structures for face perception is the fusiform face area (FFA), located in the right lateral part of the midfusiform gyrus (Kanwisher et al., 1997). Some researchers even proposed that the FFA is a specific module exclusively devoted to face recognition (Kanwisher et al., 1997; Kanwisher and Yovel, 2006). This face-specificity hypothesis contrasts with the expertise hypothesis, which maintains the FFA is a general expertise module specialized for perceptual processes associated with visual individuation (Gauthier et al., 1999, 2000). The expertise hypothesis has been tested with experts in the domains of birds (Gauthier et al., 2000), cars (Gauthier et al., 2000, 2005; GrillSpector et al., 2004; Xu, 2005), butterflies (Rhodes et al., 2004a), and novel objects classes such as greebles (Gauthier et al., 1999)

Received Oct. 31, 2010; revised April 11, 2011; accepted April 14, 2011.

Author contributions: M.B., R.L., and W.G. designed research; M.B. performed research; M.B. contributed unpublished reagents/analytic tools; M.B. analyzed data; M.B., R.L., R.U., and W.G. wrote the paper.

This work was supported by the Deutsche Forschungsgemeinschaft project GR 833/8-1 and BI 1450/1-2. We thank Michael Erb and Luca Turella for their insightful comments and help with the analysis. The help and cooperation from chess players is greatly appreciated.

The authors declare no competing financial interests.

Correspondence should be addressed to Merim Bilalić, Department of Neuroradiology, University of Tübingen, Hoppe-Seyler-Strasse 3, 72076 Tübingen, Germany. E-mail: merim.bilalic@med.uni-tuebingen.de.

DOI:10.1523/JNEUROSCI.5727-10.2011

Copyright $\odot 2011$ the authors $\quad 0270-6474 / 11 / 3110206-09 \$ 15.00 / 0$ with mixed results. In most cases, researchers sought to rule out performance-based differences by asking participants to identify isolated expertise objects or to remember their location. In contrast, Harley and colleagues (2009) looked at performance differences in identifying abnormalities in x-ray images. Although there was no difference in the FFA activation among expert and novice radiologists, the FFA activations were highly correlated with behavioral performance among experts but not among novices.

Here, we use another expertise domain, chess, to examine the expertise hypothesis. Chess involves the same basic recognition process related to individuation of single objects as is entailed in other visual domains, because players need to differentiate between objects (Saariluoma, 1995). An additional facet of chess, however, is that the objects on the board form complex spatial relationships given by rules and these rules constrain the way each piece can be moved. The most difficult aspect of the game of chess, which makes it similar to real-life decision situations, is the need to cope simultaneously with multiple objects forming numerous interrelations (Gobet and Simon, 1996a). Chess objects and their positions on the board, however, do not contain any face-specific features, making them particularly suitable for testing the face-specificity hypothesis of FFA (Kanwisher and Yovel, 2006). We exploited these features of chess and compared the face-specificity hypothesis against the more general expertise hypothesis of FFA functioning when identification of chess stimuli was necessary (experiment 1) and when more complex chessexpertise processes were required (experiments 2 and 3 ). If FFA plays a role in the perception of chess stimuli, we would expect its activation to be expertise-modulated whenever domain-specific stimuli are presented. Additionally, if FFA influences the pro- 
cesses that are generally thought to underlie chess skill (Gobet et al., 2004), we expect it to be sensitive to domain-specific task requirements.

\section{Materials and Methods}

Participants. Table 1 presents the information about the number of experts and novices, their mean age (with SD), and their chess ability score [mean Elo rating with SD; available only for experts (see Expertise measure and design, below)]. Most experts and novices participated in all three experiments (altogether, there were eight experts and nine novices). Our expert sample size corresponded to samples used in behavioral research on expertise (Bilalić et al., 2008a,b, 2009; Brockmole et al., 2008; Kiesel et al., 2009) and was larger than those in the few neuroimaging studies involving chess experts (Campitelli et al., 2005, 2007, 2008). All participants were male and right-handed. Written informed consent was obtained in line with the study protocol as approved by the Ethics Committee of Tübingen University.

Expertise measure and design. Our experts were exceptionally skilled players, rated based on their performance against other rated players. The international chess Elo scale is an interval scale with a theoretical mean of 1500 and a SD of 200. Beginners have a rating of $\sim 500$, while the best players, Grand Masters, have ratings of $>2500$. Experts are players with a rating of 2000 Elo points or more. Our experts were highly rated [ $\sim 2100$ points on average, 3 SDs above the average player (Table 1)] and were thus highly skilled chess players. Novice players were hobby players who played chess occasionally. Although novices were not rated, because they did not play chess regularly (and not in chess clubs and tournaments), it was obvious that their chess skills were vastly inferior to the experts'. In other words, experts and novices were at different ends of the same continuum (chess skill).

The expertise approach of comparing experts and novices maximizes the differences and thus provides power to capture the effects of interests despite the relatively small sample sizes intrinsic in research on experts. In a sense, the expertise approach is inherently a correlation approach. Although the use of extreme groups does not provide a quantification of skill-behavior (or skill-brain-activity) associations across the whole skill range, it tests for skill-related differences between two groups at different ends of the same continuum.

Tasks, stimuli and apparatus. The face-recognition paradigm was a localizer task used to isolate individual FFAs by having participants passively watch pictures of faces and objects (for examples of FFAs, see supplemental Fig. S1, available at www.jneurosci.org as supplemental material). The pictures of faces were taken from students at Tübingen University (Leube et al., 2001, 2003).

In experiment 1, participants indicated whether the current stimulus was the same as the previous one (one-back task). There were four classes of stimuli: chess and face stimuli, which were presented upright and inverted (Fig. 1A). The face stimuli were black-and-white pictures of students not previously used in the localizer task. The chess stimuli were full-board positions taken from a four-million-chess-games database (ChessBase Mega Base 2007; www.chessbase.com).

Experiment 2 featured the following tasks: recognizing whether the white king is in check (Check task), recognizing whether knights of either color are present (Knight task), and recognizing whether a dot of either color is presented (Dot task) (Fig. 2A). The stimuli were naturalistic; they consisted of full chess positions (containing 15-18 pieces) presented on a typical $8 \times 8$ square chess board. There were two types of positions, normal and random. The normal positions were taken from the same ChessBase database used in experiment 1 and were typical midgame positions of master games highly unlikely to have been known to participants. The random positions were generated by distributing the pieces on the board randomly using the rule that any piece of either color can occur on any square (Gobet and Waters, 2003: Vicente and Wang, 1998).

Experiment 3 also used a full chess board with 15-18 pieces presented in normal and random positions. New midgame positions were sampled from the ChessBase database. The tasks involved enumerations of chess pieces and their relations (Fig. $3 A$ ). In the Threats task, players indicated whether the number of threats (black to white) was four. In the Knights-

\section{Table 1. Participantss}

\begin{tabular}{llllll}
\hline Experiment & Group & Age \pm SD & Elo \pm SD & SDs above mean & $n$ \\
\hline I & Expert & $30 \pm 2$ & $2117 \pm 53$ & 3 & 7 \\
II & Novice & $28 \pm 1$ & - & - & 8 \\
& Expert & $30 \pm 2$ & $2117 \pm 53$ & 3 & 7 \\
III & Novice & $29 \pm 1$ & - & - & 7 \\
& Expert & $31 \pm 2$ & $2114 \pm 63$ & 3 & 6 \\
& Novice & $29 \pm 1$ & - & - & 7 \\
\hline
\end{tabular}

Group, mean age, and mean skill level as measured by the Elo rating (see Materials and Methods) with SD, number of SD above the mean, and number of players in each group in all four experiments.

and-Bishops condition, the task was to indicate whether the number of knights and bishops of both colors was four. Finally, in the non-chess Control task, all pieces regardless of color or type were counted (participants indicated whether the number is 15).

In all experiments, the stimuli were projected onto a screen above the head of the participant via a video projector in the adjacent room. The setup resulted in a visual field of $14.6^{\circ}$ for the whole scene (face or chess board). Participants saw the stimuli through a mirror mounted on the head coil and indicated their decision by pressing one of two buttons of an MRI-compatible response device held in their right hand (left button was for YES and right button for NO).

Design and procedure. All players first did the localizer task. This was comprised of two runs, each containing five blocks of faces and objects. There were 20 faces or objects in each block. Each stimulus was presented for $750 \mathrm{~ms}$ with a gap of $250 \mathrm{~ms}$ between them. The run started with a baseline (a gray screen with a black center cross), which lasted for $14 \mathrm{~s}$ and was presented after every block. Block order was randomly chosen for every participant.

In experiment 1 , we presented face or chess stimuli (upright or inverted) in blocks of five stimuli (Fig. $1 B$ ). A single stimulus lasted for $2.75 \mathrm{~s}$ and was followed by a mask. A baseline (gray screen with a center cross) was presented at the beginning, after each block, and at the end of the experiment for $14 \mathrm{~s}$. All four conditions were presented in each of the three runs four times ( 12 blocks of each condition in all runs).

We used a similar block design in experiment 2 (Fig. $2 B$ ). There were four runs with 12 blocks each [two blocks per condition (task and position type) in a single run]. The runs were block-randomized and counterbalanced across participants. The experiment started with a gray screen with a black center cross, which lasted 5-10 s, immediately followed by the instruction for $2.5 \mathrm{~s}$, after which the actual block started. The stimulus was presented for $4 \mathrm{~s}$ and was followed by a mask made of a scrambled chess position, which lasted for $0.5 \mathrm{~s}$. There were four trials (stimuli) in each block and baseline was always presented afterward.

Experiment 3 introduced a different design (Fig. 3B). There were six runs, two for each task. There was only one task (e.g., Threats task) in a single run. In one run, 10 meaningful and 10 meaningless stimuli were presented randomly. The runs were block-randomized and counterbalanced across participants. We first presented a starting board (all pieces at their initial location) with a fixation cross as a baseline with jittered duration $(6-10 \mathrm{~s})$. After a short gap (0.5 s), the target stimulus was presented until response, followed by the baseline of the next trial. Before the actual sessions, participants were given two practice trials for each task. The reaction time (i.e., the time to complete the task) was the time from when the stimulus appeared until the participant pressed the button.

Behavioral data analysis. We analyzed the behavioral data in the first experiment using a $2 \times 2 \times 2$ [expertise (experts/novices) $\times$ stimulus orientation (normal/inverted) $\times$ stimulus (chess/face)] ANOVA. Additionally, a $2 \times 2$ (expertise $\times$ stimulus orientation) ANOVA for the chess and face stimuli was conducted separately. In experiments 2 and 3 , we used $2 \times 2 \times 3$ [expertise $\times$ position type (normal/random) $\times$ task (check/knights/control)] ANOVAs. Additional $2 \times 2$ (expertise $\times$ position type) ANOVAs for each of the three tasks was conducted separately in experiments 2 and 3 . We report significant effects $(p<0.05)$ and some trends in detail the Results, below. Main effects and interactions that are not presented were not significant. 
MRI acquisition. All fMRI data were acquired using a 3-T scanner (Siemens Trio) with a 12-channel head coil. All measurements covered the whole brain using a standard echo-planar-imaging (EPI) sequence with the following parameters: TR, $2.5 \mathrm{~s}$; FOV, $192 \times 192$; TE, $35 \mathrm{~ms}$; matrix size, $64 \times 64$; 36 slices with thickness of $3.2+0.8 \mathrm{~mm}$ gap resulting in voxels with the resolution of $3 \times 3 \times 4 \mathrm{~mm}^{3}$. Finally, anatomical images covering whole brain with 176 sagittal slices were obtained after the functional runs using an MP-RAGE sequence with a voxel resolution of $1 \times 1 \times 1 \mathrm{~mm}^{3}$ (TR, $2.3 \mathrm{~s}$; TI, $1.1 \mathrm{~s}$; TE, $2.92 \mathrm{~ms}$ ).

Functional MRI data analysis. For all analyses of the fMRI data, we used the Statistical Parametric Mapping software package (SPM5; Wellcome Department of Imaging Neuroscience, London, UK; http://www.fil.ion.ucl.ac.uk/spm). All functional images were preprocessed together. This involved spatial realignment to the mean image, including unwarping, and coregistration of the mean EPI to the anatomical image for every participant. The images were neither normalized nor smoothed, since we aimed to identify the exact location of each participant's right FFA as the region of interest (ROI) using the localizer task. We therefore included blocks of faces and objects together with an implicitly modeled baseline in a general linear model. Modeling of the time series of hemodynamic activation relied on a canonical response function. Autocorrelation of the data was corrected using a first-order autoregressive model. A high-pass filter with a cutoff of $128 \mathrm{~Hz}$ was applied to eliminate low-frequency noise components. The right FFA was identified in each participant as the activated area in the right lateral part of the mid-fusiform gyrus when we subtracted activation while passively watching objects from activation while passively watching faces. In most participants, we were able to apply a stringent criterion including only voxels significant at $p<0.0001$, but in two participants, we could identify FFA only at $p<0.001$. These individually identified FFAs were used to extract the activation level in all four experiments.

In the first two experiments, we modeled all trials in their entirety; in experiment 3, we only used the first second of each trial to keep the duration for each condition constant (using the original trial durations produced similar results). The rest of the trial was explicitly specified as a nuisance regressor and the baseline was implicitly modeled. Once we specified conditions of interest, the ROI analysis was performed on the mean percentage signal change extracted using Marsbar SPM Toolbox from all the voxels within the selected region.

Control ROIs. We were primarily interested in the role of FFA in chess expertise and thus we report only the activations in the right FFA. Whole brain maps of experiments can be found in the supplemental material, available at www.jneurosci.org.

In addition to the right FFA, we identified two different sets of control ROIs to supplement our results. In the first set, we isolated another face area in every participant- the posterior part of the superior temporal sulcus (pSTS) (Campanella and Belin, 2007). In all participants, only voxels that were significantly more active when viewing faces than objects at $p<0.0001$ in the localizer task were included. Second, we isolated the intraparietal sulcus, an area subserving top-down attention (Corbetta and Shulman, 2002), to control for attentional effects in our experiments. We did this by using the one-back task (first experiment), which engages attentional processes in addition to working-memory maintenance. In all participants, we only considered voxels that were significantly more active during the one-back task (regardless of the stimuli) than during baseline at $p<0.05$ (FWE) level. Given that face processing is associated with areas in the right hemisphere (Kanwisher and Yovel, 2006), just like sustaining top-down attention (Pardo et al., 1991; Lawrence et al., 2003), we focused our analysis on right-hemisphere areas. Other face areas, such as the occipital face area, could not be isolated in most participants (there was a similar situation with the other left analogous face areas, which could not be identified in a substantial number of participants). The location and the size of the ROIs can be found in the supplemental material, available at www.jneurosci.org.

We also wanted to see whether chess expertise engages FFA exclusively as the focal area, or whether a similar pattern of results can be found in neighboring areas within the ventral cortex. We identified the peak coordinates of FFA based on the localizer group maps and extracted activations in all three experiments within the $6 \times 6 \times 6 \mathrm{~mm}^{3}$ area of the peak coordinates. We then created four additional ROIs medial, lateral, anterior, and posterior to the actual FFA by changing the values of the $x$ and $y$ coordinates for 10 or -10 (see supplemental material, available at www.jneurosci.org, for the exact coordinates in each of the ROIs) (for a similar approach, see James et al., 2005; Xue and Poldrack, 2007; Wong et al., 2009). We briefly mention the results of these analyses here. The complete analysis can be found in the supplemental material, available at www.jneurosci.org.

\section{Results}

After identifying individual right FFAs in all players using the difference in neural activation between passively watching faces and objects, these individual FFAs were used as ROIs in which we measured activation levels during the three experiments (see Materials and Methods, above). The results of the control ROIs, pSTS, and intraparietal sulcus (IPS) are briefly discussed here (for more details, see the supplemental material, available at www.jneurosci.org).

\section{Experiment 1: FFA activation in recognition of face and (naturalistic) chess stimuli}

In the first experiment, sequences of faces and naturalistic chess stimuli (a full board with chess pieces) were presented in a oneback task (Fig. 1A,B). Given that one of the hallmarks of face expertise is the impaired performance for faces presented inverted (Yin, 1969; Robbins and McKone, 2007), both chess and face stimuli were presented in upright or inverted orientation (Fig. 1A). Chess experts and novices were required to indicate whether the current picture equaled the previous one in a block design (one-back task) (Fig. 1B).

\section{Behavioral results}

Experts were generally faster than novices (ANOVA main effect of chess expertise: $F_{(1,13)}=7.2, p=0.019$ ) (Fig. 1C), but this effect was the consequence of experts' performance on chess stimuli, where they were faster than novices (ANOVA interaction: chess expertise $\times$ stimulus type: $\left.F_{(1,13)}=11, p=0.006\right)$. In general, participants recognized faces faster than chess stimuli (ANOVA main effect of stimulus type: $F_{(1,13)}=27.9, p<0.0001$ ), while the inverted orientation of stimuli generally hampered performance (ANOVA main effect of stimulus orientation: $F_{(1,13)}=$ $16.4, p=0.001)$. This was especially the case when players dealt with faces (ANOVA interaction: stimulus type $\times$ stimulus orientation: $F_{(1,13)}=8.1, p=0.014$; there was no statistically significant stimulus-orientation effect for chess stimuli: $F_{(1,13)}=2.7$, $p=0.124)$. The three-way interaction between expertise, stimulus type, and orientation was not significant.

\section{Neuroimaging results}

Face stimuli generally elicited more FFA activation than did chess stimuli (ANOVA main effect of stimulus type: $F_{(1,13)}=51, p<$ $0.001)$. Inverted stimuli elicited more activation in FFA than upright stimuli did (ANOVA main effect of stimulus orientation: $\left.F_{(1,13)}=11, p=0.004\right)$, but this effect was mainly driven by stronger FFA responses to inverted chess stimuli (ANOVA interaction task type $\times$ stimulus orientation: $\left.F_{(1,13)}=6, p=0.029\right)$. When we separately analyzed the specific stimulus types (chess or faces), we found FFA was more activated in expert players than novices when recognizing chess stimuli (planned contrast of expertise with chess stimuli: $F_{(1,13)}=5.4, p=0.037$ ) and in response to inverted chess stimuli across expertise groups (planned contrast of orientation of chess stimuli: $\left.F_{(1,13)}=23.3, p<0.001\right)$. No such effects were found for face stimuli.

The neighboring medial, lateral, anterior, and posterior ROIs to the FFA did not show expertise effects (see sup- 

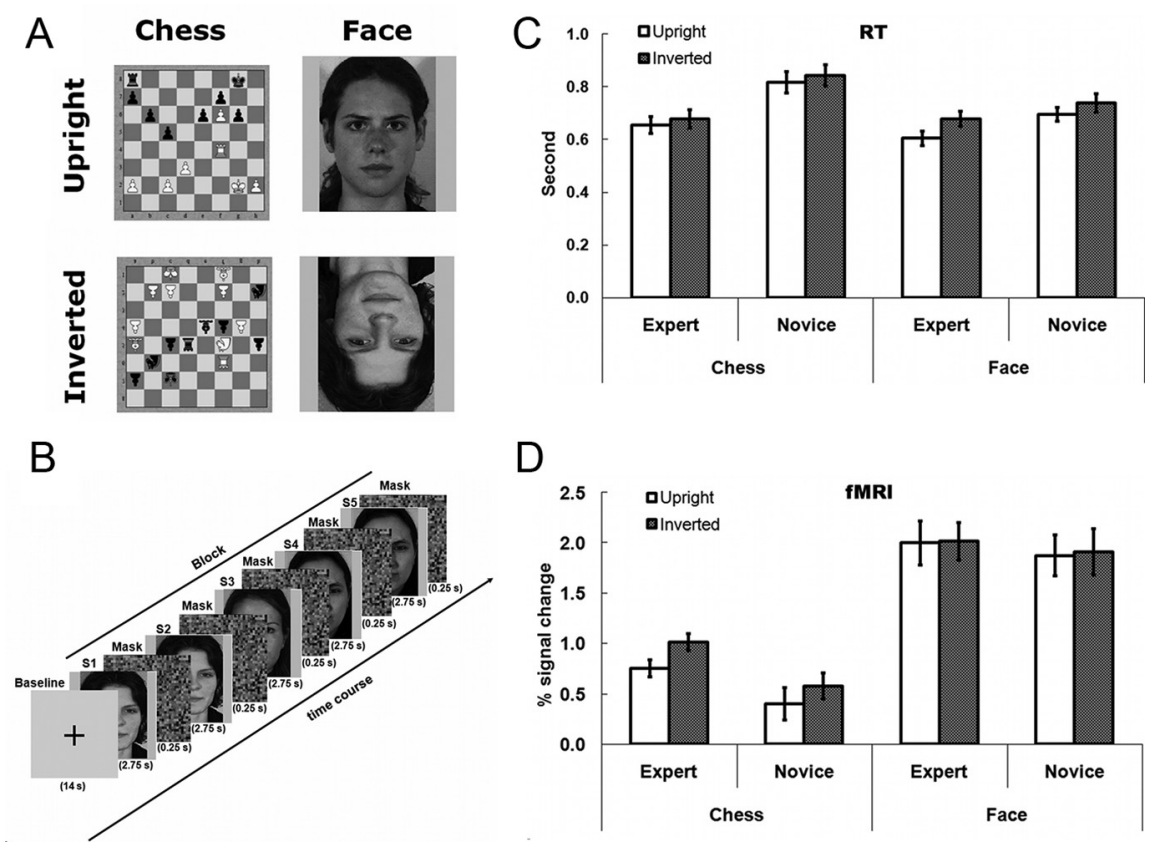

Figure 1. Experiment 1: stimuli, design, fMRI, and behavioral results. $\boldsymbol{A}$, Pictures of chess positions or student faces were presented upright or inverted. Participants had to indicate whether the currently presented stimulus matched previously presented stimulus (one-back task). $\boldsymbol{B}$, Diagram depicting the trial structure in experiment 1. There were two classes of stimuli (chess and faces) and two locations (upright and inverted), for a total of four conditions. All four conditions were presented in each of the three runs four times (12 blocks of each condition in all runs). Blocks included five stimuli (S1-S5), each lasting $1.75 \mathrm{~s}$ with a $0.25 \mathrm{~s}$ gap between them. $C$, Time (in seconds) experts and novices needed to match face and chess stimuli when they were presented upright or inverted in experiment 1. RT, Reaction time. $\boldsymbol{D}$, Activation levels (percentage signal change relative to baseline) in the right FFA in experts and novices on the chess and face stimuli depending on the location in experiment 1. Error bars indicate SEM.

plemental material, available at www.jneurosci.org). The other face-processing area, pSTS, was also more activated in both groups when dealing with faces as opposed to chess stimuli. Unlike the FFA, pSTS was not modulated by expertise when the chess stimuli were presented (see supplemental material, available at www.jneurosci.org).

\section{Experiment 2: Chess-specific expertise processes and task requirements}

We have established that faces generally elicit more activation in FFA of both experts and novices than chess stimuli. FFA responses to chess stimuli, however, were modulated by chess expertise. In the second experiment, we again used typical naturalistic chess stimuli (full $8 \times 8$ chess board and numerous pieces on it) (Fig. 2A) but added chess-specific tasks that captured skills relevant for playing chess. In the Check task, players indicated whether the white king was in check. In the second chess task (Knight task), players indicated whether the chess position contained two specific pieces (knights of either color). Both tasks required recognizing chess pieces, and the Check task additionally required recognizing the relations between the white king and potentially attacking black pieces.

The use of a naturalistic (i.e., domain-typical) setting taps additional recognition processes. To find their way through the jungle of complex relationships between multiple chess pieces, chess experts use acquired knowledge structures, called chunks (Chase and Simon, 1973) and templates (Gobet and Simon, 1996a), to direct their attention to the relevant aspects of the chess board. Just like we know by experience where the light switch is typically located in a room, expert chess players know where certain pieces can be typically found on a board full of pieces. Novices lack these highly specific knowledge structures and thus often consider irrelevant paths. Hence, the additional aspect in this third experiment is the possibility of using chess knowledge about typical places where certain pieces are found and the typical relations between them (Saariluoma, 1995).

Finally, in a non-chess control condition (Dot task) (Fig. 2A), players had to indicate whether two dots (one white, one black; size of a chess piece) were present. The dots were easily distinguishable from chess pieces, and chess knowledge should not haven given an advantage to experts, as the dots were distributed randomly on the board. In all three tasks, we used normal positions from master games and random positions where the pieces were scattered on the board (Gobet and Simon, 1996b). Although normal and random positions contained identical elements, the randomization disturbed the typical configurations of pieces and thus made the domain-specific knowledge about common positions of pieces and their relationships difficult to use (Chase and Simon, 1973; Gobet and Simon, 1996a,b; Gobet et al., 2001). This manipulation was similar to that of scattering the parts of the face (eyes, nose, mouth) within the boundaries of the face (Liu et al., 2010). Although all elements are present, the common spatial relationships between them are disrupted. If FFA is relevant for sophisticated expertise-related recognition processes, we would expect a difference between normal and random positions only in chess tasks and only in experts, since-as alluded to above-novices lack usable (i.e., chess-specific) knowledge structures.

\section{Behavioral results}

Experts were faster overall on all three tasks (ANOVA main effect of chess expertise: $F_{(1,12)}=12.1, p=0.005$ ) (Fig. $2 C$ ), but this difference was the consequence of experts' performance on the chess tasks and not on the control task (ANOVA interaction: chess expertise $\times$ task: $F_{(1,12)}=12.5, p=0.004$; planned contrast of chess expertise in the Check task: $F_{(1,12)}=16.8, p=0.001$; and in the Knight task: $\left.F_{(1,12)}=6.1, p=0.029\right)$. The tasks also differed in that the Check task appeared more difficult than the Knight task (ANOVA main effect of task: $F_{(1,12)}=339, p<0.001$ ), as indicated by longer reaction times for both experts and novices $(t$ test for dependent samples: $\left.t_{(13)}=12.2, p<0.001\right)$. Similarly, the Knight task was more difficult than the non-chess Dot task $\left(t_{(13)}=7.9, p<\right.$ 0.001 ) and consequently, the Check task was also more difficult than the Dot task $\left(t_{(13)}=13.4, p<0.001\right)$. Across groups and tasks, random positions were generally responded to more slowly, suggesting that they were generally more difficult (ANOVA main effect of chess expertise: $F_{(1,12)}=190, p<0.001$ ). The difference between normal and random positions was mainly visible on the Check tasks; there were no differences in the control Dot task (ANOVA interaction: position type $\times$ task: $F_{(1,12)}=190, p<$ 0.001 ; planned contrast of position type in the Check task: $F_{(1,12)}=284.5, p<0.001$; and Knight task: $F_{(1,12)}=28.9, p<$ $0.001)$. These effects, however, were mainly driven by the slowing 
in experts, who were significantly more impaired by the randomization of pieces (ANOVA interaction expertise $\times$ position type: $F_{(1,12)}=16.1, p=0.002$; planned contrast of chess expertise $\times$ position type interaction in the Check task: $F_{(1,12)}=$ $18.4, p=0.001$; and Knight task: $F_{(1,12)}=$ $7, p<0.021)$. In addition, this pattern of results was visible in the chess tasks but not in the control Dot task (ANOVA interaction: chess expertise $\times$ position type $\times$ task: $\left.F_{(1,12)}=23.1, p<0.001\right)$.

Although the Dot task was a non-chess task merely using a chess environment as background, experts were nevertheless faster in detecting the dots than novices (planned contrast of expertise in the Dot task: $\left.F_{(1,12)}=6.7, p=0.023\right)$. In contrast to the two chess tasks, however, the Dot task did not seem to tap expert knowledge about the typical spatial layout of chess positions, since it did not matter which position type (i.e., random or normal) was presented.

\section{Neuroimaging results}

Figure $2 D$ shows that all tasks elicited higher FFA activation in experts than in novices (ANOVA main effect of chess expertise: $F_{(1,12)}=6.9, p=0.023$; planned contrast of chess expertise in the Check task: $F_{(1,12)}=6.8, p=0.002$; planned contrast of chess expertise in the Knight task:

$F_{(1,12)}=5.3, p=0.041$; planned contrast of chess expertise in the Dot task: $\left.F_{(1,12)}=9.4, p=0.009\right)$. There were no differences between tasks (main effect task) nor between experts and novices across tasks (interaction chess expertise $\times$ task). Random positions, however, in general elicited more FFA activations than normal positions (ANOVA main effect of position type: $F_{(1,12)}=$ $69.2, p=0.011$; planned contrast of position type in the Check task: $F_{(1,12)}=14.4, p=0.003$; planned contrast of position type in the Dot task: $\left.F_{(1,12)}=3.4, p=0.089\right)$. This was a direct consequence of higher sensitivity to random piece arrangements among experts (ANOVA interaction: chess expertise $\times$ position type: $F_{(1,12)}=7.9, p=0.017$; planned contrast of chess expertise $\times$ position type interaction in the Check task: $F_{(1,12)}=8.9$, $p=0.011$; planned contrast of chess expertise $\times$ position type interaction in the Knight task: $F_{(1,12)}=2.6, p=0.13$; planned contrast of chess expertise $\times$ position type interaction in the Dot task: $\left.F_{(1,12)}=2.5, p=0.14\right)$.

The expertise effects were confined to the actual FFA, as the neighboring ROIs did not show any significant differences (supplemental material, available at www.jneurosci.org). The other face area, pSTS, did not show the same pattern of results and, generally, was not particularly responsive to the three tasks. There were no differences in pSTS activation between the tasks or between experts and novices. The attention-control area, IPS, was highly sensitive to all three tasks, in particular to the Check and Knight tasks, which were also the most difficult tasks, as indicated by the time needed for their completion (Fig. 2C). Random positions engaged IPS to a larger extent in the chess tasks (Check and Knight), indicating that their navigation may have put more demand on top-down attentional control processes than the same task with normal positions (for statistics and figures, see supplemental material, available at www.jneurosci.org). There were, however, no differences in IPS activation between experts and novices across tasks.

The lack of behavioral differences between normal and random positions, one of the hallmarks of expertise (Ericsson and Lehmann, 1996; Vicente and Wang, 1998), in the Dot task indicates that the advantage of experts in this non-chess control condition is probably unrelated to their skills relevant to playing chess. In other words, it is possible that they were more motivated or that their general recognition processes were more efficient than those of novices in this particular context. Either way, this makes it difficult to interpret the FFA activation in the control task. It is possible that the FFA activation in the control task reflects a response to automatic processes related exclusively to stimuli and not task requirements. However, the control task also needed less time to be completed than the other two tasks. Given that a single trial in a block lasted $4 \mathrm{~s}$ (Fig. $2 \mathrm{~B}$ ), participants had a chance to look at chess stimuli for almost $3 \mathrm{~s}$ once they completed the dot task. It is unclear what kind of processes were at play while players passively observed the chess stimuli. It is thus possible that the difference in FFA activation between experts and novices is a consequence of this passive observation of chess stimuli (i.e., an automatic processing of the domain-relevant stimuli) and not of the processes related to the dot search.

It is also possible that the between-group difference in FFA activation is not a consequence of the expertise-related processing differences facilitating task execution. Rather, the difference in FFA activation may reflect the fact that more complex stimuli were used. Although the differentiation between normal and random positions among experts may speak against this possibility 
A

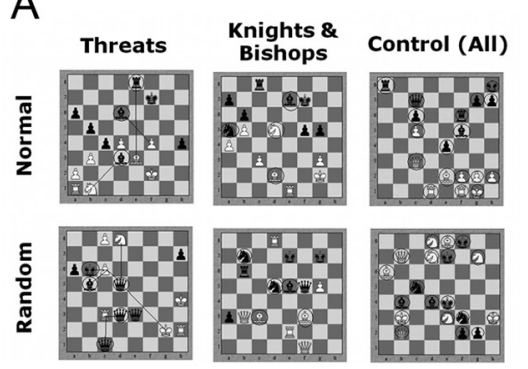

B

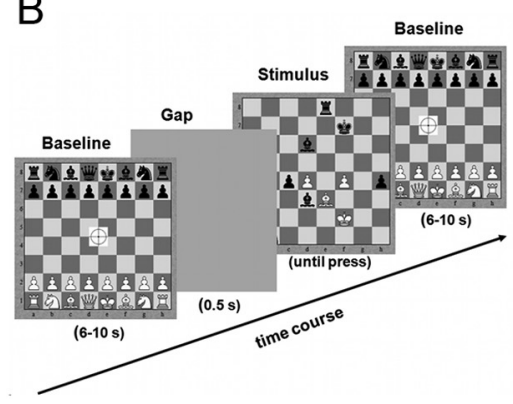

C

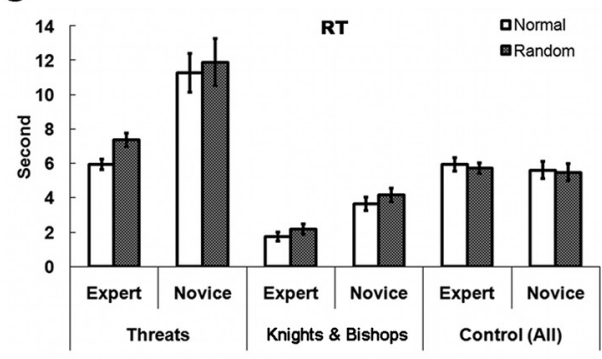

D

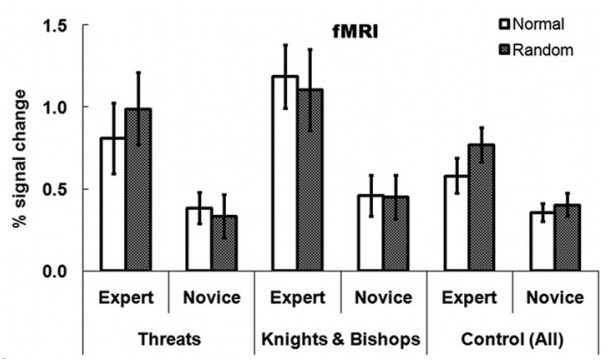

Figure 3. Experiment 3: stimuli, design, fMRI, and behavioral results. $\boldsymbol{A}$, The chess stimuli and tasks used in experiment 3 . Participants had to count the number of times black could take white pieces in the threats task, the number of knights and bishops in the knights and bishops task, and the number of all pieces on the board in the control (all) task. In all three tasks there were two types of positions: normal (taken from chess games of masters) and random (pieces were randomly distributed on the board). $\boldsymbol{B}$, Diagram depicting the trial structure in experiment 3. The baseline stimulus was an initial chess board configuration with a fixation cross; its duration was jittered. A gap in stimulus presentation was used as a warning about the upcoming stimulus. The actual chess stimulus (normal and random positions) was then presented. After the players indicated their answers by pressing one of the response buttons, the baseline stimulus of the next trial was presented. $\boldsymbol{C}$, Time (in seconds) experts and novices took to complete the threats, knights and bishops, and control tasks depending on the type of position in experiment 3. RT, Reaction time. D, Activation levels (percentage signal change relative to baseline) in the right FFA in experts and novices when completing the threats, knights and bishops, and control tasks depending on the type of position in experiment 3. Error bars indicate SEM.

(since both position types were similarly complex), a more direct test may be necessary.

\section{Experiment 3: Controlling for activity and complexity of stimuli}

The final experiment aimed to resolve remaining ambiguities from experiment 2 by using a non-chess control task designed to engage participants in chess-unrelated cognitive activity across the entire trial so as to not give them time to (passively) process the chess-related information inherent in the stimuli. Further, we used the chess starting position (a full board with all pieces at their starting location) as visual input during baseline (instead of the blank screen with fixation cross). FFA activation should thus more specifically reflect the expertise processes involved in task execution, because the difference in visual complexity between baseline and task stimuli is minimized. Experts and novices again executed two chess-related tasks and one non-chess control task (Fig. 3A). In the first chess task (Threats task), players had to count the number of threats, that is, how many times black pieces could capture white ones. This task is very similar to the previously used Check task, since check is one possible kind of threat. In the second chess task (Knights and bishops), participants had to count the number of knights and bishops. Again, this task taps the same processes as the previous Knight task, because it requires a differentiation between chess pieces. Finally, in the Control task, players counted all pieces without regard to the different kinds.

Thus, the chess-specific tasks enabled us to capture the processes of simple object detection (discrimination between differ- ent pieces) and relationship between pieces, as well as the use of more sophisticated pattern recognition processes (based on knowledge of where certain pieces are usually found). The control task did not require any of these processes but only simple foreground-background discrimination. Using the enumeration tasks made certain that participants were doing a chess-unrelated task throughout the whole trial. As in experiment 2, we also manipulated the relationship between chess pieces by presenting normal and random positions. Unlike the previous experiments, however, stimuli were presented individually and not in blocks (Fig. 3B).

\section{Behavioral results}

Experts were faster than novices across tasks (ANOVA main effect of chess expertise: $F_{(1,11)}=8.6, p=0.014$ ) (Fig. $3 C$ ), but this effect was, again, exclusively driven by differences in the chess tasks (ANOVA interaction: chess expertise $\times$ task: $F_{(1,11)}=$ 28.3; $p<0.001$; planned contrast of chess expertise in the Threats task: $F_{(1,11)}=$ $12.7 ; p=0.004$; and Knights and bishops task: $\left.F_{(1,11)}=17.9, p=0.001\right)$. All players were similarly faster with normal than random positions across tasks (ANOVA main effect of position type: $F_{(1,11)}=85.2$, $p<0.001)$. This difference, however, was exclusively driven by the chess tasks, while there was no difference in the control task (ANOVA interaction position type $\times$ task: $F_{(1,11)}=57.4, p<0.001$; planned contrast of position type in the Threats task: $F_{(1,11)}=50.8, p<0.001$; and Knights and Bishops task: $\left.F_{(1,11)}=45.7, p<0.001\right)$. These results indicate that the chess tasks benefited from chess skill, while the control task, although it also used chess stimuli, did not.

\section{Neuroimaging results}

FFA showed a similar pattern of activation as in experiment 2 (Fig. 3D). Experts had more activation in FFA than novices across all tasks (ANOVA main effect of position type: $F_{(1,11)}=8.7, p=$ 0.013; planned contrast of chess expertise in the Threats task: $F_{(1,11)}=5.6, p=0.038$; Knights and Bishops task: $F_{(1,11)}=8.2$, $p=0.015$; Control task: $\left.F_{(1,11)}=7.7, p=0.018\right)$. Despite the absence of behavioral differences in the Control task, FFA activity in general depended on expertise level. Neither were there differences in FFA activation between the three tasks, nor did experts and novices display different patterns of activity in the three tasks. Also, no global differences were found between normal and random positions across tasks and groups. Nevertheless, differences between normal and random positions were found in specific tasks (ANOVA interaction position type $\times$ task: $F_{(1,11)}=7.5, p=$ 0.019 ): there were differences (or trends) between normal and random positions in both Threats and Control tasks (planned contrast of position type in the Threats task: $F_{(1,11)}=2.3, p=$ 0.12 ; in the Control task: $\left.F_{(1,11)}=5.7, p=0.036\right)$, but there were no position-related differences in the Knights and Bishops task. The observed differences were caused by experts, whose FFA activity differed between normal and random positions, unlike novices' (ANOVA interaction: expertise $\times$ position type $\times$ task: 
$\left.F_{(1,11)}=6.6, p=0.026\right)$. Again, Threats and Control tasks exhibited trends for this pattern of results (planned contrast of expertise $\times$ position type interaction in the Threats task: $F_{(1,11)}=4.3$, $p=0.063$; in the Control task: $\left.F_{(1,11)}=2.2, p=0.17\right)$, unlike the Knights and Bishops task.

Similar to the previous experiments, FFA was the focus of the expertise effects-no significant effects were found in the neighboring ROIs (see supplemental material, available at www. jneurosci.org). The pSTS was not responsive to any of the tasks in experiment 3 , as indicated by activations among the baseline level. There were also no differences between the tasks or groups. IPS was activated during all three tasks, in particular during both chess tasks, but there were no differences between groups (see supplemental material, available at www.jneurosci.org).

\section{Discussion}

In a series of three experiments, we investigated the role of FFA in processing nonfacial stimuli, testing the face-specificity hypothesis against the more general expertise hypothesis of FFA function. In particular, we used chess stimuli presented in naturalistic or random board positions to examine FFA responses in chess experts and novices when executing mental operations related and unrelated to chess skill. Experiment 1 demonstrated that faces engage FFA more than full-board chess positions but that FFA is also modulated by expertise- experts' FFAs were more activated by chess stimuli than that of novices. The next experiments featured tasks requiring domain-specific skills. We found expertise effects in FFA in both experiments, regardless of the chess-specific activity performed. Even activities not related to chess expertise in control tasks elicited stronger activation in experts' than in novices' FFA (as long as they featured naturalistic chess stimuli). We thus showed that FFA activity is not modulated by the chess-specific task requirements but rather by the expertise-related objects presented in the domain's naturalistic context. This strongly argues against an exclusive dedication of FFA to processing faces but corroborates the more general expertise hypothesis of FFA function. The stronger FFA activity in response to faces compared with chess stimuli (cf. experiment 1 ) is also in line with the expertise hypothesis, since even highly skilled chess experts, as used in this study, will have been exposed much more often to faces than chess stimuli across their lives, making them better experts in face than chess processing.

It is difficult to explain our results solely with attentional effects. IPS, an attention-related area, was engaged in all tasks, but there were no differences between experts and novices. Some tasks, such as chess-specific tasks in experiments 2 and 3 , engaged IPS to a larger extent than control tasks, but the activation in FFA was not different between chess-related tasks. This indicates that the FFA activation was probably independent of task difficulty and the attentional processes necessary in this particular context.

Similarly, the differences in eye movements are unlikely to account for the expertise effects in FFA. It is known that experts gain advantage from focusing on different, more important aspects of chess positions (de Groot and Gobet, 1996). There are, however, usually no general differences in the number and duration of fixations. Even the differing aspects of stimuli that experts and novices attend are perceptually still the same chess objects. If these differences related to chess-playing skills modulated FFA activation, we would also expect different levels of FFA activation in control tasks when these skills were not necessary. Instead, we found no differences in FFA activation between chess-specific and chess-unrelated control tasks among experts (Figs. 2D,3D).
It also does not seem plausible that the mere complexity of stimuli, such as the size of the board or the number of objects on it, was responsible for expertise effects in FFA. If that were the case, one would expect similar activations in FFA relative to the baseline in experiment 3 where the baseline was the initial position. The activity on naturalistic game positions, where chess objects formed spatial relations, was, however, much stronger than during baseline, where these relations were absent (chess objects at initial locations do not form any meaningful relation for chess players). An additional piece of evidence that the FFA effects are not related to the mere complexity is the pattern of results on normal and random positions. Both position types are comparable in that they involve a similar number of chess objects forming interrelations on the same full chess board. Only normal positions, however, contain relational patterns between chess pieces that are meaningful to experts. FFA appears to be responsive to this subtle distinction, as shown by the different activation levels between normal and random positions among experts only.

The results from our three experiments also shed light on FFA function in complex visual domains in general. On the one hand, our findings confirm studies that reported stronger FFA activity for faces than for other objects (Kanwisher et al., 1997; GrillSpector et al., 2004; Rhodes et al., 2004a). Although experts activated FFA more than novices in matching chess stimuli (experiment 1), the response in FFA was almost twice as strong when the same players were confronted with faces. On the other hand, we found expertise effects in all experiments that featured naturalistic chess stimuli. Just like in previous research (Gauthier et al., 2000), FFA was the sole focus of this sensitivity to expertise because closely neighboring regions did not differentiate between the activity in experts and novices. Our results also indicate the expertise effect in FFA is selectively related to being confronted with naturalistic domain stimuli rather than chess-related processing. This shows that although FFA may be an important component in mediating chess expertise, it does not seem to be related to explicit chess-skill-dependent processes, which were tested in the second and third experiments.

Chess positions have certain commonalities with faces (Tarr and Cheng, 2003): their area is clearly defined by the chess board and they consist of multiple meaningful pieces, which form typical spatial relations. The fact that FFA did respond differently in experts versus novices to the full naturalistic stimuli points to a role of FFA in holistic stimulus processing (Tanaka and Farah, 1993; Gauthier and Tarr, 1997, 2002; Liu et al., 2010). An additional piece of evidence supporting this notion is the sensitivity of FFA to the disruption of typical spatial relations among chess stimuli: only experts, who are highly familiar with such relations, were affected by the configurational disruption in random positions (experiments 2 and 3 ) or when the whole board was turned upside-down (experiment 1). Unlike in some experiments using faces (Yovel and Kanwisher, 2005; Liu et al., 2010), the activation was higher with the disrupting random positions than with normal positions. The higher activation on random chess stimuli might reflect higher demands on the FFA's holistic processing resources (Henson et al., 2000).

Alternatively, this discrepancy between findings from research on faces versus chess stimuli may reflect specialization processes. While it seems the identity of faces is encoded and retrieved in FFA (Haxby et al., 2000; Lehmann et al., 2004; Winston et al., 2004; Calder and Young, 2005; Loffler et al., 2005; but see Rotshtein et al., 2005; Kriegeskorte et al., 2007; Nestor et al., 2008), we have recently shown that the utilization of chess knowledge structures-a process similar to the identification of 
faces - is related to the middle part of the collateral sulcus (Bilalic et al., 2010). The discrepancy and similarities in the specialization of the ventral visual stream for distinct stimuli such as faces and chess positions invites conjectures as to the role of FFA in visual expertise. Our results indicate that FFA is not directly related to core expertise processes but that it may support some of them indirectly by processing the stimuli holistically. The real utilization of stored chess knowledge by experts seems to be mediated by the collateral sulcus. It should be noted, though, that even in face perception, we have a dedicated network of brain structures, which are responsible for different processes (Tovée, 1998). It is thus plausible that we may have similar complex networks for processing other overlearned objects (Moore et al., 2006; Op de Beeck et al., 2006). Which areas of the ventral stream are engaged most likely depends on the nature of the stimuli. Simple stimuli (e.g., isolated chess pieces), which do not consist of complex relational patterns formed by clearly distinct individual elements, may not engage holistic processing properties of the FFA. In contrast, naturalistic multipart stimuli (e.g., faces, full-board chess positions) seem to invite holistic processing in experts, mediated by increased FFA activity.

It cannot be excluded that only parts of the FFA may be responsible for the expertise effects in our study. The FFA may not be a homogeneous area (Grill-Spector et al., 2006b; Hanson and Schmidt, 2011), and its different parts may indeed be differentially sensitive to processes associated with chess-like stimuli. Further examination with high-resolution imaging or, alternatively, with adaptation paradigms (Rhodes et al., 2004b; GrillSpector et al., 2006a) might clarify this issue.

In sum, our results reveal that brain areas-or at least parts thereof-previously assumed to be specialized modules for processing a specific category of visual stimuli (i.e., faces) may also engage in processing chess stimuli. This provides clear-cut evidence for a role of FFA in processing highly familiar non-face stimuli, supporting the more general expertise hypothesis of FFA function. Our series of experiments using visual stimuli of the face-unrelated domain of chess in combination with different levels of expertise in this domain suggests that FFA may play a role in mediating expertise through implementing the holistic processing of (naturalistic) domain stimuli. Thus, although FFA may not directly support core processes of skill use (i.e., application of probabilistic knowledge on the spatial distributions of objects), it might contribute to experts' superior performance by mediating automatic pattern generation in visual perception.

\section{References}

Bilalić M, McLeod P, Gobet F (2008a) Why good thoughts block better ones: the mechanism of the pernicious Einstellung (set) effect. Cognition 108:652-661.

Bilalić M, McLeod P, Gobet F (2008b) Inflexibility of experts: reality or myth? Quantifying the Einstellung effect in chess masters. Cognit Psychol 56:73-102.

Bilalić M, McLeod P, Gobet F (2009) Specialization effect and its influence on memory and problem solving in expert chess players. Cognit Sci 33:1117-1143.

Bilalić M, Langner R, Erb M, Grodd W (2010) Mechanisms and neural basis of object and pattern recognition: a study with chess experts. J Exp Psychol Gen 139:728-742.

Brockmole JR, Hambrick DZ, Windisch DJ, Henderson JM (2008) The role of meaning in contextual cueing: evidence from chess expertise. Q J Exp Psychol 61:1886-1896.

Calder AJ, Young AW (2005) Understanding the recognition of facial identity and facial expression. Nat Rev Neurosci 6:641-651.

Campanella S, Belin P (2007) Integrating face and voice in person perception. Trends Cogn Sci 11:535-543.

Campitelli G, Gobet F, Parker A (2005) Structure and stimulus familiarity: a study of memory in chess-players with functional magnetic resonance imaging. Span J Psychol 8:238-245.

Campitelli G, Gobet F, Head K, Buckley M, Parker A (2007) Brain localization of memory chunks in chessplayers. Int J Neurosci 117:1641-1659.

Campitelli G, Parker A, Head K, Gobet F (2008) Left lateralization in autobiographical memory: an fMRI study using the expert archival paradigm. Int J Neurosci 118:191-209.

Chase WG, Simon HA (1973) Perception in chess. Cognit Psychol 4:55-81.

Corbetta M, Shulman GL (2002) Control of goal-directed and stimulusdriven attention in the brain. Nat Rev Neurosci 3:201-215.

de Groot AD, Gobet F (1996) Perception and memory in chess. Assen: Van Gorcum.

Ericsson KA, Lehmann AC (1996) Expert and exceptional performance: evidence of maximal adaptation to task constraints. Annu Rev Psychol 47:273-305.

Gauthier I, Nelson CA (2001) The development of face expertise. Curr Opin Neurobiol 11:219-224.

Gauthier I, Tarr MJ (1997) Becoming a "Greeble" expert: exploring mechanisms for face recognition. Vision Res 37:1673-1682.

Gauthier I, Tarr MJ (2002) Unraveling mechanisms for expert object recognition: bridging brain activity and behavior. J Exp Psychol Hum Percept Perform 28:431-446

Gauthier I, Tarr MJ, Anderson AW, Skudlarski P, Gore JC (1999) Activation of the middle fusiform 'face area' increases with expertise in recognizing novel objects. Nat Neurosci 2:568-573.

Gauthier I, Skudlarski P, Gore JC, Anderson AW (2000) Expertise for cars and birds recruits brain areas involved in face recognition. Nat Neurosci 3:191-197.

Gauthier I, Curby KM, Skudlarski P, Epstein R (2005) Activity in the human fusiform gyrus reflects separate spatial frequency channels. Cognit Affect Behav Neurosci 5:222-234.

Gobet F, Simon HA (1996a) Templates in chess memory: a mechanism for recalling several boards. Cognit Psychol 31:1-40.

Gobet F, Simon HA (1996b) Recall of random and distorted positions: implications for the theory of expertise. Mem Cognit 24:493-503.

Gobet F, Waters AJ (2003) The role of constraints in expert memory. J Exp Psychol Learn Mem Cogn 29:1082-1094.

Gobet F, Lane PC, Croker S, Cheng PC, Jones G, Oliver I, Pine JM (2001) Chunking mechanisms in human learning. Trends Cogn Sci 5:236-243.

Gobet F, de Voogt A, Retschitzki J (2004) Moves in mind: the psychology of board games. Hove: Psychology.

Grill-Spector K, Knouf N, Kanwisher N (2004) The fusiform face area subserves face perception, not generic within-category identification. Nat Neurosci 7:555-562.

Grill-Spector K, Henson R, Martin A (2006a) Repetition and the brain: neural models of stimulus-specific effects. Trends Cogn Sci 10:14-23.

Grill-Spector K, Sayres R, Ress D (2006b) High-resolution imaging reveals highly selective nonface clusters in the fusiform face area. Nat Neurosci [Erratum (2007) 10:133] 9:1177-1185.

Hanson SJ, Schmidt A (2011) High-resolution imaging of the fusiform face area (FFA) using multivariate non-linear classifiers shows diagnosticity for non-face categories. Neuroimage 54:1715-1734.

Harley EM, Pope WB, Villablanca JP, Mumford J, Suh R, Mazziotta JC, Enzmann D, Engel SA (2009) Engagement of fusiform cortex and disengagement of lateral occipital cortex in the acquisition of radiological expertise. Cereb Cortex 19:2746-2754.

Haxby JV, Hoffman EA, Gobbini MI (2000) The distributed human neural system for face perception. Trends Cogn Sci 4:223-233.

Henson R, Shallice T, Dolan R (2000) Neuroimaging evidence for dissociable forms of repetition priming. Science 287:1269-1272.

James KH, James TW, Jobard G, Wong AC, Gauthier I (2005) Letter processing in the visual system: different activation patterns for single letters and strings. Cogn Affect Behav Neurosci 5:452-466.

Kanwisher N, Yovel G (2006) The fusiform face area: a cortical region specialized for the perception of faces. Philos Trans R Soc Lond B Biol Sci 361:2109-2128.

Kanwisher N, McDermott J, Chun MM (1997) The fusiform face area: a module in human extrastriate cortex specialized for face perception. J Neurosci 17:4302-4311.

Kiesel A, Kunde W, Pohl C, Berner MP, Hoffmann J (2009) Playing chess unconsciously. J Exp Psychol Learn Mem Cogn 35:292-298.

Kriegeskorte N, Formisano E, Sorger B, Goebel R (2007) Individual faces 
elicit distinct response patterns in human anterior temporal cortex. Proc Natl Acad Sci U S A 104:20600-20605.

Lawrence NS, Ross TJ, Hoffmann R, Garavan H, Stein EA (2003) Multiple neuronal networks mediate sustained attention. J Cogn Neurosci 15:1028-1038.

Lehmann C, Mueller T, Federspiel A, Hubl D, Schroth G, Huber O, Strik W, Dierks T (2004) Dissociation between overt and unconscious face processing in fusiform face area. Neuroimage 21:75-83.

Leube DT, Erb M, Grodd W, Bartels M, Kircher TT (2001) Differential activation in parahippocampal and prefrontal cortex during word and face encoding tasks. Neuroreport 12:2773-2777.

Leube DT, Erb M, Grodd W, Bartels M, Kircher TT (2003) Successful episodic memory retrieval of newly learned faces activates a left frontoparietal network. Brain Res Cogn Brain Res 18:97-101.

Liu J, Harris A, Kanwisher N (2010) Perception of face parts and face configurations: an fMRI study. J Cogn Neurosci 22:203-211.

Loffler G, Yourganov G, Wilkinson F, Wilson HR (2005) fMRI evidence for the neural representation of faces. Nat Neurosci 8:1386-1390.

Moore CD, Cohen MX, Ranganath C (2006) Neural mechanisms of expert skills in visual working memory. J Neurosci 26:11187-11196.

Nestor A, Vettel JM, Tarr MJ (2008) Task-specific codes for face recognition: how they shape the neural representation of features for detection and individuation. PloS One 3:e3978.

Op de Beeck HP, Baker CI, DiCarlo JJ, Kanwisher NG (2006) Discrimination training alters object representations in human extrastriate cortex. J Neurosci 26:13025-13036.

Pardo JV, Fox PT, Raichle ME (1991) Localization of a human system for sustained attention by positron emission tomography. Nature 349:61-64.

Rhodes G, Byatt G, Michie PT, Puce A (2004a) Is the fusiform face area specialized for faces, individuation, or expert individuation? J Cogn Neurosci 16:189-203.

Rhodes G, Jeffery L, Watson TL, Jaquet E, Winkler C, Clifford CW (2004b) Orientation-contingent face aftereffects and implications for face-coding mechanisms. Curr Biol 14:2119-2123.
Robbins R, McKone E (2007) No face-like processing for objects-ofexpertise in three behavioural tasks. Cognition 103:34-79.

Rotshtein P, Henson RN, Treves A, Driver J, Dolan RJ (2005) Morphing Marilyn into Maggie dissociates physical and identity face representations in the brain. Nat Neurosci 8:107-113.

Saariluoma P (1995) Chess players' thinking: a cognitive psychological approach. London: Routledge.

Tanaka JW, Farah MJ (1993) Parts and wholes in face recognition. Q J Exp Psychol A 46:225-245.

Tarr MJ, Cheng YD (2003) Learning to see faces and objects. Trends Cogn Sci 7:23-30.

Tovée MJ (1998) Face processing: getting by with a little help from its friends. Curr Biol 8:R317-R20.

Vicente KJ, Wang JH (1998) An ecological theory of expertise effects in memory recall. Psychol Rev 105:33-57.

Winston JS, Henson RN, Fine-Goulden MR, Dolan RJ (2004) fMRIadaptation reveals dissociable neural representations of identity and expression in face perception. J Neurophysiol 92:1830-1839.

Wong AC, Palmeri TJ, Rogers BP, Gore JC, Gauthier I (2009) Beyond shape: how you learn about objects affects how they are represented in visual cortex. PLoS One 4:e8405.

$\mathrm{Xu} Y$ (2005) Revisiting the role of the fusiform face area in visual expertise. Cereb Cortex 15:1234-1242.

Xue G, Poldrack RA (2007) The neural substrates of visual perceptual learning of words: implications for the visual word form area hypothesis. J Cogn Neurosci 19:1643-1655.

Yin RK (1969) Looking at upside-down faces. J Exp Psychol 81:141-145.

Yovel G, Kanwisher N (2004) Face perception: domain specific, not process specific. Neuron 44:889-898.

Yovel G, Kanwisher N (2005) The neural basis of the behavioral faceinversion effect. Curr Biol 15:2256-2262.

Yue X, Tjan BS, Biederman I (2006) What makes faces special? Vision Res 46:3802-3811. 